\title{
Sobre la presencia de un supuesto Haplodontheriinae (Mammalia, Toxodontidae) en el Pleistoceno tardío de la provincia de Corrientes (Argentina)
}

\section{On the presence of an alleged Haplodontheriinae (Mammalia, Toxodontidae) from the late Pleistocene of the Corrientes province (Argentina)}

\author{
Ángel R. Miño-Boilini ${ }^{1 凶}$, Alfredo E.Zurita ${ }^{1}$, Mariano Bond ${ }^{2}$, Analía Francia $^{3}$ y Esteban Soibelzon ${ }^{2}$ \\ ${ }^{1}$ Consejo Nacional de Investigaciones Cientificas y Técnicas, Centro de Ecología Aplicada del Litoral. Ruta 5, Km 2.5 (3400), Casilla de Correo 128, \\ Corrientes, Argentina. \\ ${ }^{2}$ Consejo Nacional de Investigaciones Cientificas y Técnicas, Departamento Cientifico Paleontología Vertebrados, Museo de La Plata, Universidad \\ Nacional de La Plata. Paseo del Bosque s/n (1900), La Plata, Buenos Aires, Argentina. \\ ${ }^{3}$ Agencia Nacional de Promoción Científica y Tecnológica. Centro de Ecología Aplicada del Litoral. Ruta 5, Km 2.5 (3400), Casilla de Correo 128, \\ Corrientes, Argentina. \\ 凶angelmioboilini@yahoo.com.ar
}

\begin{abstract}
Resumen. En el extremo sur de Sudamérica se han postulado varias áreas que podrían haber actuado como reservorio de taxones supérstites, especialmente en el lapso Mioceno-Pleistoceno tardío ("Araucanense"-Lujanense). Así, en el Pleistoceno tardío ( $c$. 58-28 ka) de la provincia de Corrientes, Argentina, se ha reconocido la persistencia de ciertos taxones ya desaparecidos en la región pampeana de Argentina, particularmente entre los Xenarthra Glyptodontidae, Artiodactyla Cervidae y Notoungulata "Haplodontheriinae". Aquí se efectua una revisión con criterios taxonómicos modernos de los materiales ( 3 restos dentarios) asignados a Toxodontidae Haplodontheriinae, cuyos registros últimos en la región pampeana provienen del Montehermosense (Plioceno). El estudio comparado indica que los 2 "caniniformes" superiores derechos (CTES-PZ 1608, 1609) corresponden en realidad a un Xenarthra Phyllophaga (Lestodon), en tanto que el tercero (CTES-PZ 1610) debe ser asignado a un Notoungulata (Toxodon), ambos géneros típicos del Pleistoceno de América del Sur. Por último, las revisiones sistemáticas que se llevan a cabo son claves para refinar el registro paleontológico regional, y brindar un aporte fundamental al esquema bioestratigráfico en áreas extra-pampeanas.
\end{abstract}

Palabras clave: taxonomía, taxones supérstites, América del Sur, Cuaternario.

\begin{abstract}
In southern South America, some regions have been postulated as containing supersite taxa, especially during the Miocene-late Pleistocene lapse. Thus, from the Late Pleistocene ( $c a$. 58-28 ka) of the current territory of the Corrientes Province, Argentina, it has been recognized the presence of some taxa, which were extinct from the Pampean region of Argentina: Xenarthra Glyptodontidae, Artiodactyla Cervidae and Notoungulata "Haplodontheriinae". In this contribution, we carried out a review with modern taxonomic criteria of the materials belonging to Toxodontidae "Haplodontheriinae" (represented by 3 teeth), whose last record in the Pampean region corresponds to Montehermosan Age/Stage (Pliocene). The comparative study clearly shows that 2 of the 3 teeth (CTES-PZ 1608, 1609) belong to the Xenrthra Phyllophaga (Lestodon), whereas the other (CTES-PZ 1610) corresponds to the Notoungulata (Toxodon). Both genera are typical of the Pleistocene of South America. Finally, this kind of taxonomic revisions are essentials to depurate the regional paleontological record, and thus to provide a key contribution to the biostratigraphic scheme in extra-pampean areas.
\end{abstract}

Key words: taxonomy, supersite taxa, South America, Quaternary.

\section{Introducción}

La posibilidad de que en América del Sur algunas áreas hayan actuado como reservorios de taxones supérstites ha sido postulada en distintas oportunidades. Así, durante el

Recibido: 18 mayo 2011; 26 julio 2011
Mioceno tardío-Plioceno ("Mesopotamiense"), la región mesopotámica y el noroeste del actual territorio de Argentina ("Araucanense") han sido interpretados como áreas donde se habría producido tal situación (véase Cione et al., 1999; Bossi y Gavriloff, 1998; Gavriloff, 2000). Un ejemplo frecuentemente citado, fuera del actual territorio de la Argentina, es el valle de Tarija (Bolivia), cuya fauna se asigna al Pleistoceno tardío (ca. 44-21 Ma; Coltorti et al., 
2007) y en la que Tonni et al. (2009) plantean la posibilidad de supervivencia de 2 especies con presencia exclusiva en el Piso/Edad Ensenadense (Pleistoceno Inferior a Medio; Soibelzon et al., 2008) de la región pampeana de Argentina: Arctotherium angustidens Gervais y Ameghino, 1880 (Carnivora, Ursidae) y Glyptodon munizi Ameghino, 1881 (Xenarthra, Glyptodontidae). Posiblemente dicha continuidad local se deba a causas ecológicas o históricas propias de esa región (véase también Soibelzon et al., 2005; Zurita et al., 2009).

En Argentina, para el Pleistoceno, una de las áreas donde se ha observado este posible escenario ha sido la región mesopotámica, y más particularmente en el Pleistoceno tardío (Piso/Edad Lujanense), de la provincia de Corrientes (formaciones Toropí y Yupoí; ca. 58-28 ka; véase Tonni, 2009) con la supuesta persistencia de taxones de antigüedad Montehermosense (Plioceno), Ensenadense (Pleistoceno Inferior a Medio) y Bonaerense (Pleistoceno Medio a Superior) (Álvarez, 1974; Carlini et al., 2004, 2008). Recientemente se ha comprobado que para ciertos taxones estos supuestos casos de supervivencia se deben, en realidad, a un desconocimiento de la distribución estratigráfica real de los grupos, más que a una persistencia en la paleofauna. Así, se ha observado que taxones como Scelidodon Ameghino, 1881 (Xenarthra, Phyllophaga) poseen un biocrón mayor al originalmente postulado, que se consideraba solamente limitado al Piso/Edad Ensenadense de la región pampeana (Miño-Boilini y Carlini, 2009); su registro en el piso/edad Lujanense (Pleistoceno tardío) de la provincia de Corrientes es una evidencia de su presencia sin implicar necesariamente un caso de supervivencia como fuera inicialmente postulado (Gasparini y Zurita, 2005 y las referencias allí citadas).

En este contexto, Álvarez (1974) registró un caso particular representado por la persistencia de algunos taxones pampeanos en el Pleistoceno de la provincia de Corrientes, más precisamente en el área del Arroyo Toropí (283' S y $59^{\circ} 02^{\prime}$ O), Departamento de Bella Vista, provincia de Corrientes. Entre los materiales hallados y descriptos, mencionó restos dentarios de un Notoungulata Toxodontidae atribuido a "Haplodontheriinae" (sensu Kraglievich, 1934), un grupo de toxodontes de gran porte y que se caracterizaba por la presencia de un abultamiento craneano del frontal (e.g. Trigodon), que indicaría la presencia de un cuerno. Los toxodontidos "haplodonterinos", actualmente incluidos dentro de la subfamilia Toxodontinae (Nasif et al., 2000), tenían sus últimos registros en el Piso/Edad Montehermosense (Mioceno-Plioceno) de la región pampeana (Bond et al., 1995). Junto con los restos del probable "haplodontherino", Álvarez (1974) también mencionaba la presencia de un Proterotheriidae (Litopterna), un grupo que hasta ese momento (excepto una discutida cita para el Pleistoceno (Bonaerense) de Córdoba y otras muy dudosas para el Plioceno-Pleistoceno ("Puelchense") de la provincia de Buenos Aires), se consideraba extinguido en el Chapadmalalense (Plioceno temprano) (Bond, 1999; Villafañe et al., 2006). En relación con esto, Marshall et al. (1984: 29) cuestionan estas interpretaciones y sugieren 2 alternativas para explicar dicha presencia: 1), estos grupos de ejemplares se extendieron temporalmente en el Cuaternario de Corrientes y 2), los ejemplares proceden en realidad de la Formación Ituzaingó (asignada al Plioceno por Herbst y Santa Cruz, 1999), unidad que infrayace a las formaciones Toropí y Yupoí.

Posteriormente, este problema fue retomado por Bond (1986, 1999) y Bond et al. (1995) como un probable caso de persistencia de taxones montehermosenses de la región pampeana en el Pleistoceno tardío de la provincia de Corrientes, pero sin llegar a conclusiones definitivas. Sin embargo, posteriormente el hallazgo de restos de Proterotheriidae en el Pleistoceno superior de Uruguay y nuevos hallazgos en el Pleistoceno de Córdoba (Argentina), en un contexto estratigráfico preciso, permitieron constatar que los proterotéridos persistieron hasta el Pleistoceno tardío, pero fuera del área típicamente pampeana (Bond et al., 2001). De este modo, los supuestos restos de un toxodonte "haplodonterino" en la Formación Yupoí quedaban como los únicos que indicaban la posible supervivencia de un taxón montehermosense en el área mesopotámica. Debido a las consecuencias bioestratigráficas y paleobiogeográficas que ello suponía, el objetivo de este trabajo fue llevar a cabo un reestudio de los materiales asignados por Álvarez (1974) a Toxodontidae "Haplodontheriinae" a fin de intentar una aclaración de tal situación. El esquema bioestratigráfico seguido corresponde al de Cione y Tonni (2005).

\section{Materiales y métodos}

Colecciones paleontológicas donde se encuentra el material estudiado. CTES-PZ, Colección de Paleontología de la Facultad de Ciencias Exactas y Naturales y Agrimensura de la Universidad Nacional del Nordeste, Corrientes, Argentina; MLP, División Paleontología Vertebrados, Facultad de Ciencias Naturales y Museo de La Plata (La Plata, Buenos Aires); MRSC, Museo Paleontológico Real de San Carlos "Armando Calcaterra" (Colonia, Uruguay); I-i, incisivos superiores e inferiores, respectivamente.

Procedencia geográfica, marco geológico y estratigráfico de los ejemplares estudiados.

Los ejemplares estudiados (CTES-PZ: 1608, 1609, 1610) corresponden a restos dentarios fragmentarios provenientes de la Formación Yupoí (Herbst, 1971), del área del Arroyo

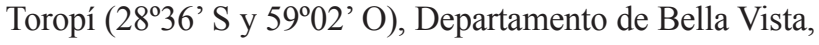
provincia de Corrientes, Argentina (Fig. 1). 
Inicialmente, desde un punto de vista geológico, Álvarez (1974) reconoció para esta área una única unidad litoestratigráfica, la Formación Yupoí, que incluía 2 miembros (superior e inferior). Posteriormente, Herbst y Álvarez (1977) y Herbst y Santa Cruz (1985), dieron caracteres de formación a estos dos miembros (formaciones Toropí y Yupoí). A partir del reestudio de los mamíferos fósiles del área, Scillato-Yané et al. (1998) asignaron a la secuencia completa una antigüedad Lujanense s.l. (Cione y Tonni, 1995, Pleistoceno medio- Holoceno temprano). Por último, fechamientos recientes de los sedimentos portadores mediante análisis OSL (Optically Stimulated Luminescence) arrojaron edades entre los 58000 y 28000 AP., esto es, Lujanense s. s.t. (Pleistoceno tardío-Holoceno temprano, Tonni et al., 2009).

Discusión y reasignación sistemática de los materiales atribuidos a un Toxodontidae "Haplodontheriinae" (Apéndice 1)

Los restos mencionados por Álvarez (1974) como pertenecientes a un toxodóntido haplodonterino corresponden a 3 dientes, 2 fragmentados y 1 más completo: CTES-PZ 1608, una porción de diente caniniforme superior derecho (Fig. 2. 1, 2); CTES-PZ 1609, gran parte de un diente caniniforme superior derecho algo fracturado (Fig. 2. 3, 4) y CTES-PZ 1610 (Fig. 2. 5, 6), extremo distal de un i3 derecho. El nuevo estudio de estos restos permitió reconocer que no corresponden en su totalidad a un Notoungulata Toxodontidae, sino que parte de estos elementos dentarios son referibles a un Phyllophaga (Xenarthra) asignable al género Lestodon; el otro resto se asigna a un Notoungulata Toxodontidae del género Toxodon. En consecuencia, la referencia a un toxodóntido de tipo "haplodonterino" y su supervivencia en el Pleistoceno superior de la Mesopotamia argentina debe ser, al menos en este caso, descartado. La descripción de los materiales y las consideraciones que llevaron a su reasignación taxonómica se hallan a continuación en la referencia de cada uno de los materiales al taxón asignado.

\section{Descripción}

Xenarthra Cope, 1889

Tardigrada Latham y Davies (en Forster), 1795

Mylodontidae Gill, 1872

Mylodontinae Gill, 1872

Lestodon Gervais, 1855

Especie Tipo. Lestodon armatus Gervais, 1855

Lestodon sp. (Fig. 2. 1, 4)

Material referido. CTES-PZ 1608, extremo distal de un caniniforme superior derecho (Fig. 2. 1, 2). CTES-PZ 1609 , extremo distal de un caniniforme superior derecho algo fracturado (Fig. 2. 3, 4).

Comentarios. Ambos caniniformes son de sección transversal subtriangular a subelíptica (Fig. 2. 2). En vista lateral,

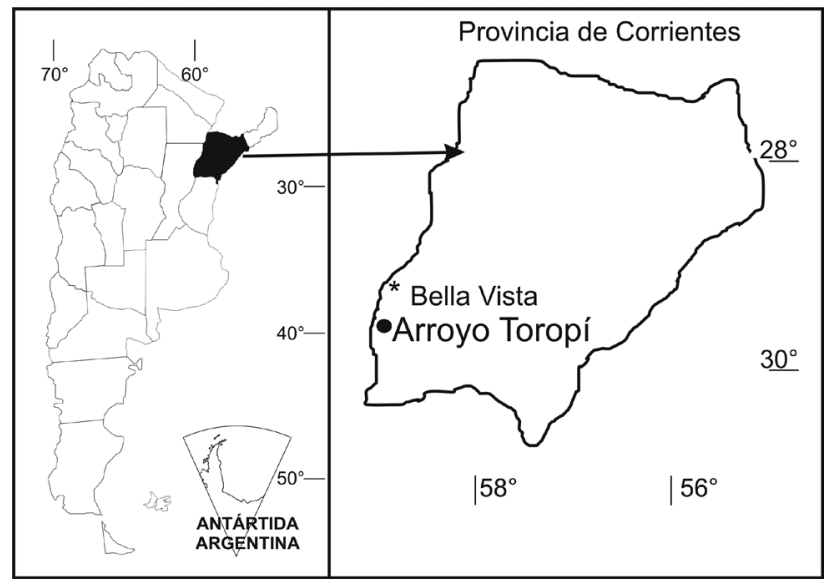

Figura 1. Ubicación del sitio paleontológico. Corrientes, Argentina.

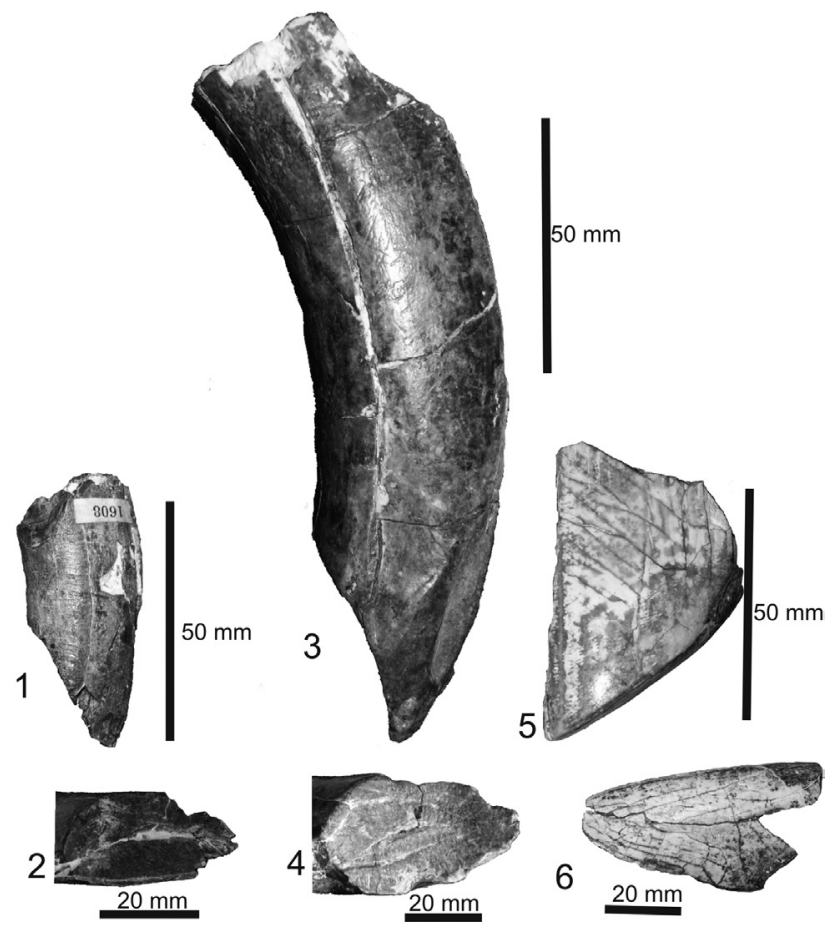

Figura 2. Caniniforme superior derecho de Lestodon sp. CTESPZ 1608 (1) vista labial y (2) vista oclusal; caniniforme superior derecho de Lestodon sp. CTES-PZ 1609 (3) vista labial y (4) vista oclusal; i3 derecho de Toxodon sp. CTES-PZ 1610 (5) vista lingual y (6) vista oclusal. Upper right caniniform of Lestodon sp. CTES-PZ 1608 (1) labial view and (2) oclussal view; upper right caniniform of Lestodon sp. CTES-PZ 1609 (3) labial view and (4) oclussal view; right i3 of Toxodon sp. CTES-PZ 1610 (5) lingual view and (6) oclussal view.

muestran su superficie oclusal claramente oblicua. Ninguno de los 2 ejemplares descriptos presenta esmalte, lo que evidentemente marca una diferencia notable con cualquier 
incisivo de un Notoungulata Toxodontidae; además, esto no puede ser atribuido a un proceso tafonómico. La ausencia de esmalte es característico para la casi totalidad de los Xenarthra (Vizcaíno, 2009). En vista oclusal, los 2 caniniformes presentan el ángulo antero-lingual redondeado y agudo, la cara distal levemente plana y las mesiales convexas (Fig. 2. 3), sin surcos visibles sobre su superficie (Fig. 2. 1). Dichos caracteres fueron mencionados por Esteban (1996) como exclusivos de los caniniformes de Lestodon. A su vez, la cara interna posee una delgada capa de cemento, visible a simple vista; otro rasgo también mencionado por Esteban (1996) para dicho taxón. Es importante señalar que el caniniforme (CTES-PZ 1609) es de mayor tamaño y se encuentra más completo (Fig. 2. 4), mostrando una evidente curvatura sobre su eje longitudinal.

Según Esteban (1996), los caniniformes de Lestodon presentan un amplio rango de variación en el que se pueden reconocer 2 tipos, de acuerdo con su sección transversal: $a$ ), los de sección transversa grande (e.g. CTES-PZ 1609) y b), los de sección transversa reducida (e.g. CTES-PZ 1608).

Por lo expuesto, se descarta la asignación de los materiales CTES-PZ 1608 y 1609 a un notoungulado Toxodontidae "Haplodontheriinae". Esto se fundamenta en la ausencia de esmalte y por su mayor ancho. En los toxodóntidos haplodontherinos (Trigodon gaudryi del Montehermosense), los I1 e I2, además de poseer una clara y visible banda de esmalte, son más comprimidos, en tanto que los incisivos inferiores son triangulares, comprimidos y procumbentes (Pascual, 1965; Pascual et al., 1966).

Orden Notoungulata Roth, 1903

Familia Toxodontidae Gervais, 1847

Género Toxodon Owen, 1837

Especie Tipo. Toxodon platensis Owen, 1837.

Toxodon sp. (Fig. 2. 5, 6)

Material referido. CTES-PZ 1610, extremo distal de un i3 derecho.

Descripción y comparación. Incisivo con la superficie oclusal de sección prismático-triangular (Fig. 2. 6), cortada a bisel lateralmente. Presenta una cara lingual y dos caras, mesial y labial, observándose una banda de esmalte sobre dichas caras. Estos caracteres fueron mencionados por Ameghino $(1887,1889)$ y Roth (1898) como propios de Toxodon; además, una comparación del material en cuestión con un i3 de Toxodon (e.g. CTES-PZ. 7218) muestra una similitud casi completa.

\section{Discusión}

Durante el Pleistoceno tardío, en el área de la Mesopotamia argentina, persisten algunos taxones que en el área pampeana parecen haber desaparecido. Ejemplo de ello son el Proterotheriidae Neolicaphrium Frenguelli, 1921 y el Cervidae Antifer ultra Ameghino, 1889. Neolicaphrium se registra en la región pampeana únicamente en el Chapadmalalense (Plioceno), con la especie N. major Soria, 2001 (véase Villafañe et al., 2006); ya en el Pleistoceno se encuentran exclusivamente fuera de la región pampeana (Bond et al., 2001), lo que según Villafañe et al. (2006), podría deberse a una extinción local en la región pampeana. En el Pleistoceno, Neolicaphrium se presenta con su especie tipo, $N$. recens Frenguelli, 1921, que se registra en sedimentos del Ensenadense y Lujanense, pero siempre fuera del ámbito de la región pampeana, o en la periferia de la misma, como en el actual territorio de la provincia de Córdoba (Tauber, 2000) y en las provincias de Corrientes, Santa Fe y Uruguay (Bond et al., 2001; Ubilla et al., 2011); representa uno de los escasos taxones de ungulados nativos que llegan hasta el Pleistoceno. A su vez, A. ultra (Artiodactyla, Cervidae), registrado en el Bonaerense de la región pampeana (Cione y Tonni, 2005), es un taxón que persiste en el Lujanense de las provincias de Entre Ríos (Formación Arroyo Feliciano) (Alcaraz et al., 2005) y Corrientes (Formación Toropí) (Alcaraz, com. pers).

El nuevo estudio de los 3 ejemplares asignados por Álvarez (1974) a un notoungulado Toxodontidae "Haplodontheriinae", que sugería la presencia de un taxón supérstite en el área mesopotámica, indica que los mismos corresponden a un Xenarthra Phyllophaga (Lestodon) y a un Notoungulata Toxodontidae (Toxodon). Cabe destacar que ambos géneros cuentan con registros previos en el Pleistoceno tardío de las provincias de Corrientes (Scillato-Yané et al., 1998; Miño-Boilini et al., 2006; Carlini et al., 2008) y Entre Ríos (Noriega et al., 2004; Ferrero et al., 2007).

Por último, las revisiones sistemáticas que se llevan a cabo son clave para los fines de refinar y depurar el registro paleontológico regional y brindan un aporte fundamental al esquema bioestratigráfico en áreas extra-pampeanas. Así como el reestudio de los ejemplares CTES-PZ 1608, 1609 y 1610 evidenció una asignación taxonómica errónea, la presencia del Glyptodontidae Panochthus cf. intermedius (un taxón limitado al Ensenadense de la región pampeana; véase Carlini y Scillato-Yané, 1999; Soibelzon et al., 2010) en el Pleistoceno tardío de Corrientes debe ser reanalizada, ya que sería probable cierta vinculación con aquellas formas intertropicales de Glyptodontidae Panochthini (Porpino y Bergqvist, 2002).

\section{Agradecimientos}

Al Dr. E. P. Tonni y a la Lic. B. B. Álvarez por las sugerencias efectuadas al trabajo. Esta contribución fue parcialmente financiada a través de los proyectos PICTOUNNE 00164 y F-011 (UNNE). 


\section{Literatura citada}

Alcaraz, M. A., B. S. Ferrero y J. I. Noriega. 2005. Primer registro de Antifer ultra Ameghino 1889 (Artiodactyla: Cervidae) en el Pleistoceno de Entre Ríos. INSUGEO (Misceláneas) 14:6570.

Álvarez, B. B. 1974. Los mamíferos fósiles del Cuaternario de Arroyo Toropí, Corrientes, Argentina. Ameghiniana 11:295311.

Ameghino, F. 1887. Observaciones generales sobre el orden de los mamíferos extinguidos llamados toxodontes (Toxodontia) y sinopsis de los géneros y especies hasta ahora conocidos. Anales del Museo de La Plata 1:1-66.

Ameghino, F. 1889. Contribución al conocimiento de los mamíferos fósiles de la República Argentina. Academia Nacional de Ciencias de Córdoba, Actas 6:1-1027.

Bond, M. 1986. Los ungulados fósiles de Argentina: evolución y paleoambientes, $4^{\circ}$ Congreso Argentino de Paleontología y Bioestratigrafía, Actas 2:173-185.

Bond, M. 1999. Quaternary native ungulates of southern South American. A synthesis. Quaternary of South America and Antarctic Peninsula 12:177-205.

Bond, M., E. Cerdeño y G. López, G. 1995. Los ungulados nativos de América del Sur. In Evolución climática y biológica de la región pampeana durante los últimos cinco millones de años. Un ensayo de correlación con el Mediterráneo occidental, M. T. Alberdi, G. Leone y E.T. Tonni (eds.). Museo Nacional de Ciencias Naturales/Consejo Superior de Investigaciones Científicas. Madrid. p. 259-275.

Bond, M., D. Perea, M. Ubilla y A. Tauber. 2001. Neolicaphrium recens Frenguelli, 1921, the only surviving Proterotheriidae (Litopterna, Mammalia) into South American Pleistocene. Paleovertebrata 30:37-50.

Bossi, G. E. y I. J. C. Gavriloff. 1998. Terciario: estratigrafía, bioestratigrafía y paleogeografía. In Geología de Tucumán., Publicación especial, M. Gianfransisco, M. E. Puchulu, J. Durango de Cabrera y G. F. Aceñolaza (eds.). Colegio de Graduados de Ciencias Geológicas de Tucumán, Tucumán. p. 87-108.

Carlini, A. A. y G. J. Scillato-Yané. 1999. Evolution of Quaternary Xenarthrans (Mammalia) of Argentina. Quaternary of South America and Antarctic Peninsula 12:149-175.

Carlini, A. A., A. E. Zurita, G. M. Gasparini y J. I. Noriega. 2004. Los mamíferos del Pleistoceno de la Mesopotamia argentina y su relación tanto con aquellos del centro-norte de la Argentina, Paraguay, sur de Bolivia, como con los del sur de Brasil y oeste de Uruguay: paleobiogeografía y paleoambientes. INSUGEO (Misceláneas) 12:5-12.

Carlini, A. A., A. E. Zurita y A. R. Miño-Boilini. 2008. Reseña paleobiogeográfica de los Xenarthra (Mammalia) del Pleistoceno tardío de la región mesopotámica (Argentina). INSUGEO (Miscelánea) 17:259-270.
Cione, A. L. y E. P. Tonni. 2005. Bioestratigrafía basada en mamíferos del Cenozoico superior de la provincia de Buenos Aires, Argentina $16^{\circ}$ Congreso Geológico Argentino (La Plata), Actas 11:183-200.

Cione, A. L., E. P. Tonni, M. Bond, A. A. Carlini, U. F. Pardiñas, G. J. Scillato-Yané, D. Verzi, y M. G. Vucetich. 1999. Ocurrence charts of Pleistocene mammals in the Pampean area, eastern Argentina. Quaternary of South America and Antarctic Peninsula 12:53-59.

Coltorti, M., L. Abbazzi, M. Ferreti, P. Lacumin, F. Paredes Ríos, M. Pellegrini, P. Pieruccini, M. Rustioni, G. Tito y L. Rook. 2007. Last Glacial Mammals in South America: a new scenario from the Tarija Basin (Bolivia). Naturwissenschaften 94:288-299.

Esteban, G. I. 1996. Revisión de los Mylodontinae cuaternarios (Edentata-Tardigrada) de Argentina, Bolivia y Uruguay. Sistemática, filogenia, paleobiología, paleozoogeografía y paleoecología. Universidad Nacional de Tucumán, Tesis, Doctorado Facultad de Ciencias Naturales e Instituto Miguel Lillo, Tucumán. 234 p.

Ferrero, B. S., D. Brandoni, J. I. Noriega y A. A. Carlini. 2007. Mamíferos de la Formación El Palmar (Pleistoceno tardío) de la provincia de Entre Ríos, Argentina. Revista del Museo Argentino de Ciencias Naturales n.s. 9:109-117.

Gasparini, G. M. y A. E. Zurita. 2005. Primer registro fósil de Tayassu pecari (Link) (Mammalia, Artiodactyla) en la Argentina. Ameghiniana 42:473-480.

Gavriloff, I. J. C. 2000. Las edades mamíferos como unidades cronoestratigráficas: la problemática del valle de Santa María, noroeste argentino. Ameghiniana Suplemento 37:9-10 R.

Herbst, R. 1971. Esquema estratigráfico de la provincia de Corrientes, República de Argentina. Revista de la Asociación Geológica Argentina 26:221-246.

Herbst, R. y J. N. Santa Cruz. 1999. Mapa litoestratigráfico de la provincia de Corrientes. D'Orbignyana 2:1-69.

Kraglievich, L. 1934. La antigüedad pliocena de las faunas de Monte Hermoso y Chapadmalal, deducidas de su comparación con las que le precedieron y sucedieron. El Siglo Ilustrado, Montevideo. $133 \mathrm{p}$.

Marshall, L., A. Berta, R. Hoffstetter, R. Pascual, O. Reig, M. Bombin y A. Mones. 1984. Mammals and stratigraphy: geochronology of the continental mammal-bearing Quaternary of South America. Palaeovertebrata, Mémoire Extraordinaire. p. 1-76.

Miño-Boilini, A. R. y A. A. Carlini. 2009. The Scelidotheriinae Ameghino, 1904 (Phyllophaga, Xenarthra) from the Ensenadan-Lujanian Stage/Ages (Early Pleistocene to EarlyMiddle Pleistocene-Early Holocene) of Argentina. Quaternary International 210:93-101.

Miño-Boilini, A. R., E. Cerdeño y M. Bond. 2006. Revisión del género Toxodon Owen, 1837 (Notoungulata: Toxodontidae) en el Pleistoceno de las provincias de Corrientes, Chaco y Santa 
Fe. Revista Española de Paleontología 21:93-103.

Nasif, N., L. S. Musalem y E. Cerdeño. 2000. A new toxodont from the late Miocene of Catamarca, Argentina, and a phylogenetic analysis of the Toxodontidae. Journal of Vertebrate Paleontology 20:591-600.

Noriega, J. I., A. A. Carlini y E. P. Tonni. 2004. Vertebrados del Pleistoceno tardío de la cuenca del arroyo Ensenada (departamento Diamante, provincia de Entre Ríos, Argentina). INSUGEO (Misceláneas) 12:71-76.

Pascual, R. 1965. Los Toxodontidae (Toxodonta, Notoungulata) de la Formación Arroyo Chasicó (Plioceno inferior) de la provincia de Buenos Aires. Características geológicas. Ameghiniana 4:101-132.

Pascual, R., E. J. Ortega-Hinojosa, D. G. Gondar y E. P. Tonni. 1966. Vertebrata. In Paleontografía Bonaerense. Vertebrata I, A. V. Borrello (ed.). Comisión de Investigaciones Científicas de la provincia de Buenos Aires 202. p.

Porpino, K. O. y L. P. Bergqvist. 2002. Novos achados de Panochthus (Mammalia, Cingulata, Glyptodontoidea) no nordestedoBrasil. Revista BrasileiradePaleontologia4:51-62.

Prevosti, F. J., A. Dondas y F. I. Islas. 2004. Revisión del registro de Theriodictis Mercerat, 1891 (Carnivora, Canidae) y descripción de un nuevo ejemplar de Theriodictis platensis Mercerat, 1891. Ameghiniana 41:245-250.

Roth, S. 1898. Catálogo de los mamíferos fósiles conservados en el Museo de La Plata. Grupo Ungulata, orden Toxodontia. Revista del Museo de La Plata 8:33-160.

Soibelzon, L. H., E. P. Tonni y M. Bond. 2005. The fossil record of South American short-faced bears (Ursidae, Tremarctinae). Journal of South American Earth Sciences 20:105-113.

Soibelzon, E., E. P. Tonni y J. C. Bidegain. 2008. Cronología, magnetoestratigrafía y caracterización bioestratigráfica del Ensenadense (Pleistoceno inferior-medio) en la ciudad de Buenos Aires. Revista de la Asociación Geológica Argentina 63:421-429.

Soibelzon, E., A. R. Miño-Boilini, A. E. Zurita y C. M. Krmpotic. 2010. Los Xenarthra (Mammalia) delEnsenadense(Pleistoceno Inferior a Medio) de la región pampeana (Argentina). Revista
Mexicana de Paleontología 27:449-469.

Scillato-Yané, G. J., E. P. Tonni, A. A. Carlini y J. I. Noriega. 1998. Nuevos hallazgos de mamíferos del Cuaternario en el arroyo Toropí, Corrientes, Argentina. Aspectos Bioestratigráficos, Paleoambiental y Paleozoogeograficos. $10^{\circ}$ Congreso Latinoamericano de Geología y $6^{\circ}$ Congreso Nacional de Geología Económica, Actas I. p. 263-268.

Tauber, A. 2000. Hallazgos de Proterotheriidae (Mammalia, Litopterna) en el Pleistoceno de Córdoba, Argentina. Ameghiniana 37:157-162.

Tonni, E.P. 2009. Las unidades portadoras de vertebrados del Cuaternario de las regiones mesopotámica y pampeana oriental de la Argentina: Ensayo de correlación. Quaternário do RS: Integrando Conhecimiento. Sociedad Brasileira de Paleontologia 57-66.

Tonni, E. P., E. Soibelzon, A. L. Cione, A. A. Carlini, G. J. Scillato Yané, A. E. Zurita y F. Paredes-Ríos. 2009. Mammals from the pleistocene of the Tarija Valley (Bolivia). Correlation with the pampean chronological standard. Quaternary International 210:57-65.

Ubilla, M., D. Perea, M. Bond y A. Rinderknecht. 2011. The first cranial remains of the Pleistocene proterotheriid Neolicaphrium recens Frenguelli, 1921 (Mammalia Litopterna): a comparative approach. Journal of Vertebrate Paleontology 31:193-201.

Villafañe, A. L., E. Ortiz-Jaureguizar y M. Bond. 2006. Cambios en la riqueza taxonómica y en las tasas de primera y última aparición de los Proterotheriidae (Mammalia, Litopterna) durante el Cenozoico. Estudios Geológicos 62:155-166.

Vizcaíno, S. F. 2009. The teeth of the "toothless": novelties and key innovations in the evolution of xenathrans (Mammalia, Xenarthra). Paleobiology 35:343-366.

Zurita, A. E., A. R. Miño-Boilini, E. Soibelzon, A. A. Carlini y F. Paredes-Ríos. 2009. The diversity of Glyptodontidae (Xenarthra, Cingulata) in the Tarija valley (Bolivia): systematic, biostratigraphic and paleobiogeographic aspects of a particular assemblage. Neues Jahrbuch für Geologie und Paläontologie-Abhandlungen 251:225-237.

Apéndice 1. Materiales de comparación.

CTES-PZ s/n. Lestodon sp.; cráneo y mandíbula. Pleistoceno tardío. Corrientes, Argentina.

MLP 3-3. Lestodon armatus; cráneo y mandíbula. Pleistoceno. Buenos Aires, Argentina.

MLP 3-30. Lestodon armatus; cráneo y mandíbula. Pleistoceno tardío. Buenos Aires, Argentina.

MRSC 1020. Lestodon sp; cráneo y mandíbula. Pleistoceno. Bahía de Colonia, Uruguay.

MRSC 520, 1097. Lestodon sp.; caniniformes aislados. Pleistoceno. Bahía de Colonia, Uruguay.

CTES-PZ 7218. Toxodon platensis; mandíbula completa. Pleistoceno tardío, Barranqueras, Chaco, Argentina. 